\title{
Formule di maggiorazione connesse ad una classe di trasformazioni lineari $\left({ }^{*}\right)$. \\ Memoria di Gamtano Fromera (a Trieste).
}

Sunto. - Si costruisce un funsionale $F(w)$ i cui valovi sono limitati inferiormente dal modulo di una trasformazione lineare, che verifica opportune ipotesi. Come applicazione vengono ottenute formole di magginrazione globale per le soluzioni di talune equacioni differenziali lineari.

Siano $S$ ed $S^{\prime}$ due spazi di Barach lineari rispetto allo stesso corpo numerico reale $N\left({ }^{1}\right)$ e sia

$$
u^{\prime}=T(u)
$$

una trasformazione lineare di $S$ in una varietà lineare $T(S)$ di $S^{\prime}$. Dicendo che la trasformanione è lineare, intendo che:

$$
T(a u+b v)=a T(u)+b T(v)
$$

quali si siano i due punti $u$ e $v$ di $S$ e i due numeri $a$ e $b$ di $N$.

Supponiamo che esista la trasformazione inversa della (1):

$$
u=T^{-1}\left(u^{\prime}\right)
$$

ovviamente lineare, e che sia continua.

Scopo del presente lavoro è di far conoscere, in opportune ipotesi per la trasformazione data e la sua inversa, un metodo per la maggiorazione del modulo della (2), cioè un procedimento per ottenere valori per eccesso dell' estremo superiore $\mu\left(T^{-1}\right)$ del funzionale:

$$
\frac{\left\|T^{-1}\left(u^{\prime}\right)\right\|}{\left\|u^{\prime}\right\|} \quad\left[u^{\prime} \subset T(S)-\omega^{\prime}, \omega^{\prime}=\text { origine di } S^{\prime}\right] .
$$

La classe di trasformazioni che considereremo include quella relativa ad una categoria di trasformazioni connesse con problemi al contorno per equazioni differenziali lineari, fra i quali rientrano quelli relativi ai classici problemi al contorno per le equazioni del secondo ordine di tipo ellittico e di tipo parabolico.

(*) Lavoro eseguito per l'IN AC nell'Istituto matematico dell' Universita di Trieste. I risultati di esso, furono comunicati al Congresso della Società Matematica Austriaca a Salisburgo (9-15 settembre 1952) ed esposti in una conferenza, tenuta presso l'Istituto Matematico dell' Università di Napoli (4 maggio 1953).

(1) G. Frchera, Lezioni sulle trasformazioni lineari, vol. I, Istituto Matematico, Universita di Trieste.

Annali di Motemation 
L'interesse, anche pratico, delle formule di maggiorazione, ottenute nel presente lavoro, risiede nella possibilita, che esse offrono, di fornire una limitazione per l'errore di approssimazione, relativo a procedimenti di calcolo delle soluzioni di equazioni lineari $\left({ }^{2}\right)$.

Per quanto riguarda le equazioni differenziali del tipo anzidetto, credo che venga per la prima volta risoluto in modo esauriente il problema della maggiorazione dell'errore relativo all'applicazione del classico metodo d'approssimazione, cosidetto dei minimi quadrati.

Si perverrà, fra l'altro, a portare un ulteriore contributo ad un risultato di Renato Caccioppoli, relativo ad una limitazione globale per le soluzioni delle equazioni differenziali lineari di tipo ellittico $\left({ }^{3}\right)$.

1. 'Teoremi generali. - Siano $B_{1}, B_{2}, \ldots, B_{n} ; G_{1}, G_{2}, \ldots, G_{m}, n+m$ insiemi boreliani rispettivamente appartenenti agli spazi euclidei $S_{k_{1}}, S_{k_{2}}, \ldots$, $S_{k_{n}} ; S_{h_{1}}, S_{h_{2}}, \ldots, S_{h_{m}}$.

Sia $S_{B}$ una varieta lineare, rispetto al corpo reale $N$, di vettori $u \equiv\left(u_{1}, u_{2}, \ldots, u_{1}\right)$ ad $n$ componenti reali di cui la $i$-esima sia definita in $B_{i}$ ed $S_{G}$ una varieta lineare di vettori $U \equiv\left(U_{1}, U_{2}, \ldots, U_{m}\right)$ con $U_{j}$ (reale) definita in $G_{j}$. Nella varietà lineare $S_{B}^{(0)}$ contenuta in $S_{B}$ siano definite le tra. sformazioni lineari :

$$
w=E(u), \quad w=E^{*}(u)
$$

aventi codomini :

$$
E\left(S_{B}^{(0)}\right) \quad \text { e } \quad E^{*}\left(S_{B}^{(0)}\right)
$$

contenuti in $S_{B}$ e contenenti $S_{B}^{(0)}$. Siano anche definite in $S_{B}^{(0)}$ le trasformagioni lineari :

$$
U=L(u), \quad U=L^{*}(u), \quad U=M(u), \quad U=M^{*}(u)
$$

con codomini :

$$
L\left(S_{B}^{(0)}\right), \quad L^{*}\left(S_{B}^{(0)}\right), \quad M\left(S_{B}^{(0)}\right), \quad M^{*}\left(S_{B}^{(0)}\right)
$$

contenuti in $S_{G}$.

Supporremo che in $B_{t}$ e in $G_{j}$ siano definite le due funzioni completamente additive non negative d'insieme $\beta_{i}\left(E_{i}\right)$ e $\gamma_{,}\left(I_{j}\right)$ per tutti gli insiemi boreliani $E_{i}$ ed $I_{j}$ rispettivamente contenuti in $B_{i}$ e $G_{j}$.

(2) Cfr. G. Fichera, loc. cit (1), pp. 250-253.

(3) Cfr. R. Caccroppol, Isimitazioni per le soluzioni di una equazione lineare ellittica a devivate parziali, *Giornale di Matematiche di Battaglini ", vol. 80, pp. 186.212, (1950.51). 
I vettori $u$ di $S_{B}$ e $U$ di $S_{G}$ siano tali che per ognuno di essi esistano finiti gli integrali di Lebesque-Streltues:

$$
\int_{B_{i}} u_{i}^{2} d \beta_{i}, \quad \int_{B_{j}} U_{j}^{2} d \gamma_{j} .
$$

Porremo, detti $u$ e $v$ vettori di $S_{B}$ :

$$
(u, v)=\sum_{i=1}^{n} \int_{B_{i}} u_{i} v_{i} d \beta_{i}
$$

e detti $U$ e $V$ due vettori di $S_{G}$ :

$$
(U, V)=\sum_{j=1}^{m} \int_{G_{j}} U_{j} V_{j} d \gamma_{j} .
$$

Epperò $S_{B}$ ed $S_{G}$ sono spazi hilbertiani.

Faremo la seguente prima ipotesi fondamentale:

$1^{\text {a) }}$ Assunti comunque due vettori $u$ e $v$ in $S_{B}^{(0)}$ sussiste l' identità:

$$
\left(u, E^{*}(v)\right)-(v, E(u))=\left(M^{*}(u), L^{*}(v)\right)-(M(v), L(u)) .
$$

Sia $S^{\prime}$ lo spazio hilbertiano $S_{B} \times S_{G}$ il cui elemento generico indicheremo con $u^{\prime} \equiv\{u, U\}$.

Consideriamo le due trasformazioni lineari di $S \equiv S_{B}^{(0)}$ in $S^{\prime}$ :

$$
u^{\prime}=T(u) \equiv\{E(u), L(u)\} ; \quad u^{\prime}=T^{*}(u) \equiv\left\{E^{*}(u), L^{*}(u)\right\}
$$

e facciamo la seguente ipotesi fondamentale :

$\left.2^{a}\right)$ Esistono le trasformazioni inverse della $T(u)$ e della $T^{*}(u)$

$$
u=T^{-1}\left(u^{\prime}\right), \quad u=T^{*-1}\left(u^{\prime}\right)
$$

Indicato con $\omega$ l'elemento nullo in $S_{B} \Theta$ con $\mathbf{Q}$ quello in $S_{G}$, poniamo:

$$
u_{1}^{\prime} \equiv\{u, \Omega\}, \quad u_{2}^{\prime} \equiv\{\omega, U\},
$$

per modo che

$$
u^{\prime}=u_{1}^{\prime}+u_{2}^{\prime}
$$

Poniamo inoltre:

$$
\begin{array}{ll}
E^{-1}(u)=T^{-1}\left(u_{1}^{\prime}\right), & L^{-1}(U)=T^{-1}\left(u_{2}^{\prime}\right) \\
E^{*-1}(u)=T^{*-1}\left(u_{1}^{\prime}\right), & L^{*-1}(U)=T^{*-1}\left(u_{2}^{\prime}\right)
\end{array}
$$

e quindi :

$$
\begin{aligned}
& T^{-1}\left(u^{\prime}\right)=E^{-1}(u)+L^{-1}(U), \\
& T^{*-1}\left(u^{\prime}\right)=E^{*-1}(u)+L^{*-1}(U) .
\end{aligned}
$$


Considereremo la seguente terza ipotesi fondamentale:

$3^{\text {a) }} I l$ codominio $L\left(S_{B}^{(0)}\right)$ di $L$ è una base per $S_{G}\left({ }^{4}\right)$.

Avvertiamo che indicheremo con $E_{i}(u)(i=1,2, \ldots, n), L_{j}(u), M_{j}(u)$ $(j=1,2, \ldots, m)$ le componenti, rispettivamente, di $E(u), L(u)$ ed $M(u)$.

Analogamento con $E_{i}^{-1}(u)$ e $L_{i}^{-1}(u)$ indicheremo le componenti di $E^{-}(u)$ ed $L^{-1}(u)$.

Sussiste il seguente teorema:

I. - Il funzionale :

$$
I(u)=\frac{\|u\|^{2}}{\|T(u)\|^{2}}=\frac{\|u\|^{2}}{\|E(u)\|^{2}+\|L(u)\|^{2}}
$$

sia dotato di massimo in $S_{B}^{(0)}-\omega$ ed esista un vettore $u_{0}$ massimante $I(u)$ tale che tutte le componenti del vettore $T\left(u_{0}\right)$ siano non negative.

Sia $\Gamma$ l'insieme, supposto non vuoto, dei vettori $w$ di $S_{B}^{(0)}$ per ognuno dei quali si ha:

$$
E^{*}(w) \subset S_{B}^{(0)}, \quad L^{*}(w)=\Omega, \quad E_{i} E^{*}(w)>0, \quad L_{j} E^{*}(w)>0 .
$$

Detto $F(w)$ il maggiore fra gli $n+m$ numeri:

$$
\underset{B_{i}}{\operatorname{estr.sup} .} \frac{w_{i}}{E_{i} E^{*}(w)}, \quad \text { estr. sup. } \frac{-M_{j}(w)}{L_{j} E^{*}(w)},
$$

se sono soddisfatte le ipotesi $\left.\left.1^{\mathrm{a}}\right), 2^{\mathrm{a}}\right)$, $3^{\mathrm{a}}$, si $h a$ :

e quindi:

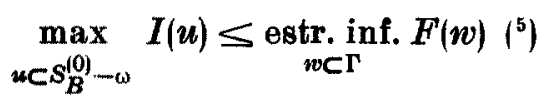

$$
\|u\|^{2} \leq F(v)\left[\|E(u)\|^{2}+\|L(u)\|^{2}\right] .
$$

Per ogni $u \subset S_{B}^{(0)}$ consideriamo il vettore $v$ verificante le equazioni :

$$
E^{*}(v)=u, \quad L^{*}(v)=\Omega
$$

tale vettore esiste ed è unico, avendo noi ammesso che la $T^{*} \dot{e}$ dotata di trasformazione inversa e che $E^{*}\left(S_{B}^{(0)}\right) \supset S_{B}^{(0)}$.

Anzichè considerare il funzionale $I(u)$ definito in $S_{B}^{(0)}-\omega$, potremo considerare il funzionale:

$$
J(v)=\frac{\left\|E^{*}(v)\right\|^{2}}{\left\|E E^{*}(v)\right\|^{2}+\left\|L E^{*}(v)\right\|^{2}},
$$

definito in $E^{*-1}\left(S_{B}^{(0)}\right)-\omega$ - Esisterà un vettore $v_{0}$ massimante $J(v)$, tale che le funzioni $E_{i} E^{*}\left(v_{0}\right)(i=1,2, \ldots, n), L_{j} E^{*}\left(v_{0}\right)(j=1,2, \ldots, m)$ siano tutte non negative.

(4) Cid significa che $L\left(S_{B}^{(0)}\right)$ è denso in $S_{G}$. Cfr. loc. cit. (1), pag. 28.

(5) Si conviene, eventualmente, di attribuire al funzionale $F(w)$, definito in $r$, anche il valore $+\infty$ in corrispondenza a qualche $w$. 
Detto $v$ un qualsiasi fissato vettore $\neq \omega$ della varieta lineare $E^{*-1}\left(S_{B}^{(0)}\right)$ e considerata la funzione di $\varepsilon: J\left(v_{0}+\varepsilon v\right)$, la condizione:

$$
\left[\frac{d J\left(v_{a}+\varepsilon v\right)}{d \varepsilon}\right]_{\varepsilon=0}=0
$$

fornisce :

$$
\begin{gathered}
\left(E^{*}(v), E^{*}\left(v_{0}\right)\right)\left(\left\|E E^{*}\left(v_{0}\right)\right\|^{2}+\left\|L E^{*}\left(v_{0}\right)\right\|^{2}\right)= \\
=\left\|E^{*}\left(v_{0}\right)\right\|^{2}\left[\left(E E^{*}(v), E E^{*}\left(v_{0}\right)\right)+\left(L E^{*}(v), L E^{*}\left(v_{0}\right)\right)\right] .
\end{gathered}
$$

Posto:

si ha:

$$
\mu=\max _{u \subset s_{B}^{(0)}-\omega} I(u)
$$

$$
\left(E^{*}(v), E^{*}\left(v_{0}\right)\right)=\mu\left[\left(E E^{*}(v), E E^{*}\left(v_{0}\right)\right)+\left(L E^{*}(v), L E^{*}\left(v_{0}\right)\right)\right] .
$$

Dalla (1) si ha d'altra parte, tenendo presente che $L^{*}(v)=L^{*}\left(v_{0}\right)=\mathbf{Q}$ :

$$
\left(E^{*}(v), E^{*}\left(v_{0}\right)\right)=\left(v_{0}, E E^{*}(v)\right)-\left\{M\left(v_{0}\right), L E^{*}(v) \mid .\right.
$$

Da (3) e (4) si trae

$$
\left(v_{0}-\mu E E^{*}\left(v_{0}\right), E E^{*}(v)\right)=\left(M\left(v_{0}\right)+\mu L E^{*}\left(v_{0}\right), L E^{*}(v)\right) .
$$

Sia $u$ un vettore di $S_{B}^{(0)}$ soluzione delle equazioni

$$
E(u)=v_{0}-\mu E E^{*}\left(v_{\mathrm{n}}\right), \quad L(u)=\mathbf{Q} .
$$

Tale vettore certo esiste in virtù dell' ipotesi $2^{a}$ ) $\theta$ dato che

$$
v_{0} \subset E^{*-1}\left(S_{B}^{(0)}\right) \subset S_{B}^{(0)} \subset E\left(S_{B}^{(0)}\right) ; \quad E E^{*}\left(v_{0}\right) \subset E\left(S_{B}^{(0)}\right) .
$$

Dalla (5) si trae, ponendovi $v=E^{*-1}(u)$

e quindi :

$$
\left\|v_{0}-\mu E E^{*}\left(v_{0}\right)\right\|=0
$$

$$
v_{0}-\mu E E^{*}\left(v_{0}\right)=\omega .
$$

Si ha allora, detto $u$ un qualsiasi vettore di $S_{B}^{(0)}$ :

$$
\left(M\left(v_{0}\right)+\mu L E^{*}\left(v_{0}\right), L(u)\right)=0
$$

ma essendo (3a ipotesi) $L\left(S_{B}^{(0)}\right)$ una base per $S_{G}$, deve essere :

$$
W=M\left(v_{0}\right)+\mu L E^{*}\left(v_{0}\right)=\Omega \text {. }
$$

Infatti, dato arbitrariamente $\varepsilon>0$, deve esserci un $u_{\varepsilon} \subset S_{B}^{(0)}$ tale che:

$$
\left\|W-L\left(u_{\varepsilon}\right)\right\|<\frac{\varepsilon}{\|W\|} .
$$

Ne segue:

$$
\|W\|^{*}=\left(W, W-L\left(u_{\varepsilon}\right) \leq\|W\|\left\|W-L\left(u_{\varepsilon}\right)\right\|<\varepsilon .\right.
$$


Abbiamo cosi dimostrato che il vettore $v_{*}$ massimante $J(v)$ è soluzione del seguente sistema:

$$
\left\{\begin{array}{l}
v_{0}-\mu E E^{*}\left(v_{0}\right)=\omega, \\
M\left(v_{0}\right)+\mu L E^{*}\left(v_{0}\right)=\Omega .
\end{array}\right.
$$

Sia ora $w$ un qualsiasi vettore appartenente a $\Gamma$. Consideriamo in corrispondenza le funzioni :

$$
f_{i}=\mu-\frac{w_{i}}{E_{i} E^{*}(w)}
$$

Supponiamo vi sia un punto $x_{i}$ in uno dei $B_{i}$ in cui $f_{i} \leq 0$. Sarà allora:

$$
\text { estr. inf. } f_{i}=\mu-\text { estr. sup. } \frac{w_{i}}{E_{i} E^{*}(w)} \leq 0
$$

e quindi $F(w) \geq \mu$.

Supponiamo che in ogni $B_{i}$ riesca $f_{i}>0$.

Indicando con $v_{0}$ il vettore massimante, sopra specificato, e detto $B_{i}^{\prime}$ l'insieme dei punti di $B_{i}$ in cui $E_{i} E^{*}\left(v_{0}\right)=0$ (e quindi anche $v_{0 i}=0$ ), si ha in $B-B_{i}^{\prime}$ :

$$
f_{i}=\frac{v_{0 i}}{E_{i} E^{*}\left(v_{0}\right)}-\frac{w_{i}}{E_{i} E^{*}(w)}=\frac{v_{0 i} E_{i} E^{*}(w)-w_{i} E_{i} E^{*}\left(v_{0}\right)}{E_{i} E^{*}\left(v_{0}\right) \cdot E_{i} E^{*}(w)}
$$

e quindi :

$$
\int_{B_{i}}\left[v_{0 i} E_{i} E^{*}(w)-w_{i} E_{i} E^{*}\left(v_{0}\right)\right] d \beta_{i} \geq 0
$$

Ne segue:

$$
\left(v_{0}, E E^{*}(w)\right)-\left(w, E E^{*}\left(v_{0}\right)\right) \geq 0 .
$$

Si ha d'altra parte:

$$
\left(v_{0}, E E^{*}(w)\right)-\left(E^{*}(w), E^{*}\left(v_{0}\right)\right)+\left(E^{*}(w), E^{*}\left(v_{0}\right)\right)-\left(w, E E^{*}\left(v_{0}\right)\right)=
$$

$=-\left(M^{*} E^{*}(w), L^{*}\left(v_{0}\right)\right)+\left(M\left(v_{0}\right), L E^{*}(w)\right)+\left(M^{*} E^{*}\left(v_{0}\right), L^{*}(w)\right)-\left(M(w), L E^{*}\left(v_{0}\right)\right)$

e quindi, ricordando che $L^{*}\left(v_{0}\right)=L^{*}(w)=\Omega$, per la (7) si trae:

$$
\left(M\left(v_{0}\right), L E^{*}(w)\right)-\left(M(w), L E^{*}\left(v_{0}\right)\right) \geq 0 .
$$

Si considerino ora le funzioni :

$$
g_{j}=\mu+\frac{M_{g}(w)}{L_{j} E^{*}(w)} .
$$

Si ha in $G_{j}-G_{j}^{\prime}$, essendo $G_{j}^{\prime}$ l'insieme in cui si annullano $M_{j}\left(v_{0}\right)$ e $L_{j} E^{*}\left(v_{0}\right)$ :

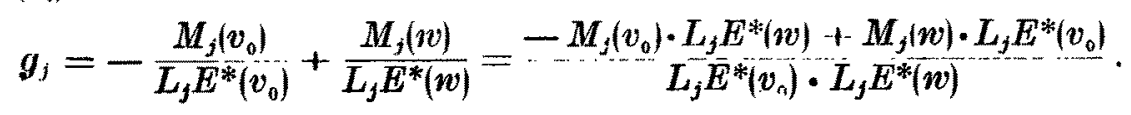


Ma in virtù della (8) deve essere :

$$
\sum_{j=1}^{m} \int_{G_{j}}\left[M_{j}(w) L_{j} E^{*}\left(v_{n}\right)-M_{j}\left(v_{0}\right) L_{j} E^{*}(w)\right] d \gamma_{j} \leq 0
$$

e quindi vi sarà almeno un indice $j$ per il quale:

$$
\int_{G_{j}}\left[M_{j}(w) L_{j} E^{*}\left(v_{0}\right)-M_{j}\left(v_{0}\right) L_{j} E^{*}(w)\right] d \gamma_{j} \leq 0 .
$$

Ciò implica, essendo $L_{j} E^{*}\left(v_{n}\right) \cdot L_{j} E^{*}(w) \geq 0$, che in qualche punto $x_{j}$ di $G_{j}-G_{j}^{\prime}$ sarà $g_{j} \leq 0$.

Ne segue:

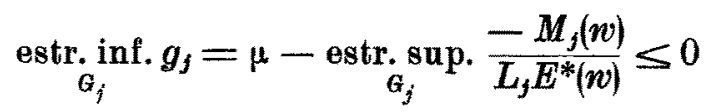

e quindi :

$$
\mu \leq F(w)
$$

Il teorema è così completamente dimostrato.

In modo perfettamente analogo si dimostra il seguente:

II. - Il funzionale $I(u)$ sia dotato di minimo in $S_{B}^{(0)}-\omega$ ed esista un vettore $u_{0}$ minimante $I(u)$ tale che tutte le componenti del vettore $T\left(u_{0}\right)$ siano non negative.

Sia $\Gamma$ l'insieme dei vettori $w$ di $S_{B}^{(0)}$ indicato nell' enunciato del teorema precedente.

Detto $F(w)$ il minore fra gli $n+m$ numeri:

$$
\underset{B_{i}}{\operatorname{estr} \text { inf. }} \frac{w_{i}}{E_{i} E^{*}(w)}, \quad \text { estr. inf. } \frac{-M_{j}(w)}{L_{j} E^{*}(w)},
$$

se sono soddisfatte le ipotesi $\left.\left.\left.1^{\mathrm{a}}\right), 2^{\mathrm{a}}\right), 3^{\mathrm{a}}\right)$ si ha:

$$
\|u\|^{2} \geq F(w)\left[\|E(u)\|^{2}+\|L(u)\|^{2}\right]
$$

La dimostrazione è perfettamente analoga a quella del teorema I.

Evidentemente perchè questo ultimo teorema sia espressivo, occorrerà supporre che non sia vuoto il sottoinsieme di $\Gamma$ costituito dagli elementi $w$ pei quali :

$$
w_{i}>0 \quad(i=1,2, \ldots, n), \quad M_{f}(w)<0 \quad(j=1,2, \ldots, m) .
$$

Per quanto riguarda l'esistenza del massimo del funzionale $I(u)$, sussiste il seguente teorema:

III. - Se il codominio $T(S)$ della trasformazione $T$ è una varietà com. pleta dello spazio $S^{\prime}$ e se la forma quadratica $\left\|T^{-1}\left(u^{\prime}\right)\right\|^{2}$, definita in $T(S)$, è totalmente continua, esiste il massimo di $I(u)$ in $S_{B}^{(0)}$. 
Per la dimostrazione di questo teorema rimandiamo alle «Lezioni sulle trasformazioni lineari $》$ citate nella nota ( $\left.{ }^{1}\right)$ (cfr. pag. 220) $\left({ }^{5}\right)$.

Analogamente sussiste il teorema:

IV. - Se $S$ è uno spazio hilbertiano completo e se la forma quadratica $\|T(u)\|^{2}$ è totalmente continua, esiste $i$ minimo di $I(u)$ in $S_{B}^{(0)}$.

Circa il segno delle componenti del vettore massimante (minimante) $I(u)$, il seguente teorema fornisce una condizione sufficiente perchè esse abbiano tutte lo stesso segno. $E\left(S_{R}^{01}\right)$ :

V. - Se esistono una trasformazione di $S_{B}$ in una sua parte contenente

$$
w=\varphi(u)
$$

ed una trasformazione di $S_{G}$ in una sua parte, contenente $L\left(S_{B}^{(0)}\right)$ :

tali che :

$$
W=\Phi(U)
$$

$$
\begin{aligned}
& 0 \leq w_{i} \leq\left|u_{i}\right|, \quad 0 \leq W_{j} \leq\left|U_{j}\right|, \quad\left|E_{i}^{-1}[\varphi(u)]+L_{i}^{-1}[\Phi(U)]\right| \geq\left|E_{i}^{-1}(u)+L_{i}^{-1}(u)\right| \\
& {\left[w_{i} \geq\left|u_{i}\right|, \quad W_{i} \geq\left|U_{j}\right|, \quad\left|E_{i}^{-1}[\varphi(u)]+L_{i}^{-1}[\Phi(U)]\right| \leq\left|E_{i}^{-1}(u)+L_{i}^{-1}(u)\right|\right]} \\
& (i=1,2, \ldots, n ; j=1,2, \ldots, m)
\end{aligned}
$$

e se $I(u)$ è dotato di massimo (minimo) in $S_{B}^{(0)}$, allora vi è un vettore massi. mante (minimante) $I(u)$ che ha le componenti tutte dello stesso segno.

La dimostrazione segue ovviamente dall'osservare che si ha:

$$
I(u) \leq \frac{\left\|E^{-1}[\varphi(u)]+L{ }^{1}[\Phi(U)]\right\|^{2}}{\|\varphi(u)\|^{2}+\|\Phi(U)\|^{2}}
$$

(o la diseguaglianza opposta nel easo del minimo).

Vogliamo osservare che il teorema I si può generalizzare nel seguente:

VI. - Il funzionale $I(u)$ sia dotato di massimo in $S_{B}^{(0)}-\omega$ ed esista un vettore $u_{0}$ di esso massimante, tale che il vettore $T\left(u_{0}\right) \equiv\left(E\left(u_{0}\right), L\left(u_{0}\right)\right)$ abbia non negative le componenti $E_{1}\left(u_{n}\right), E_{2}\left(u_{0}\right), \ldots, E_{n}\left(u_{0}\right) ; L_{1}\left(u_{n}\right), L_{2}\left(u_{0}\right), \ldots, L_{m}\left(u_{0}\right)$ $\left(n^{\prime} \leq n, m^{\prime} \leq m\right)$ e non positive le altre.

Sia $\Gamma$ l'insieme, supposto non vuoto, dei vettori $w$ di $S_{R}^{(11)}$ per ognuno dei quali si ha:

$$
\begin{array}{rlrl} 
& E^{*}(w) \subset S_{B}^{(0)}, & L^{*}(w)=9 \\
E_{t} E^{*}(w)>0 & \left(i=1,2, \ldots, n^{\prime}\right), & E_{k} E^{*}(w)<0 & \left(k=n^{\prime}+1, \ldots, n\right) \\
L_{j} E^{*}(w)<0 & \left(j=1,2, \ldots, m^{\prime}\right), & L_{h} E^{*}(w)<0 & \left(h=m^{\prime}+1, \ldots, m\right) .
\end{array}
$$

(6) Cfr. anche F. Riusz - B. S. Nagr. Leçons $d^{\prime}$ Analyse fonctionelle, Acadímie des Sciences de Hongrie, Burlapost, 1952. 
Detto $F(v)$ il maggiore fra gli $n+m$ numeri:

$$
\underset{B_{i}}{\operatorname{estr.~sup.}} \frac{w_{i}}{E_{i} E^{*}(w)}, \quad \text { estr. sup. } \frac{-M_{j}(w)}{L_{j} E^{*}(w)},
$$

se sono soddisfatte le ipotesi $\left.\left.1^{\mathfrak{a}}\right), 2^{\mathrm{a}}\right)$, $3^{\mathrm{a}}$ ) si ha:

$$
\max _{u \subset S_{B}^{(0)}-\omega} I(u) \leq \underset{w \subset \Gamma}{\text { estr. inf. } F(w) .}
$$

La dimostrazione è perfettamente identica a quella del teorema $\mathrm{I}$.

Analoga generalizzazione sussiste per il teorema II.

Nelle applicazioni può presentarsi non agevole il verificare che il funzionale del teorema $I$ è dotato di massimo. Può riuscire allora utile sostituire il teorema $I$ con il seguente:

VII. - Il funzionale $I(u)$ sia limitato in $S-\omega$ e dettone $\mu$ l'estremo superiore esista una successione $\left\{v^{(k)}\right\}$ verificante le seguenti condizioni:

a)

$$
\begin{gathered}
L\left(v^{(k)}\right)=\Omega ; \quad E^{*}\left(v^{(k)}\right) \subset S_{B}^{(0)} \\
H\left(v^{(k)}\right)=\sum_{i=1}^{n} \int_{B_{i}}\left|E_{i} E^{*}\left(v^{(k)}\right)\right| d \beta_{i}+\sum_{j=1}^{m} \int_{G_{j}}\left|L_{j} E^{*}\left(v^{(k)}\right)\right| d \gamma_{j}<+\infty
\end{gathered}
$$

b)

$$
\operatorname{Max}_{k \rightarrow \infty} \lim \left(v^{(k)}\right)>0 ;
$$

$$
\operatorname{Max}_{k \rightarrow \infty} \lim _{k \rightarrow \infty}\left(\left\|E E^{*}\left(v^{(k)}\right)\right\|^{*}+\left\|L E^{*}\left(v^{(k)}\right)\right\|^{2}\right)<+\infty .
$$

c)

$$
\begin{gathered}
E_{i} E^{*}\left(v^{(k)}\right) \geq 0 ; \quad L_{j} E^{*}\left(v^{(h)}\right) \geq 0, \\
(i=1,2, \ldots, n ; j=1,2, \ldots, m) ; \\
\lim _{k \rightarrow \infty} J\left(v^{(k)}\right)=\lim _{k \rightarrow \infty} \frac{\left\|E^{*}\left(v^{(k)}\right)\right\|^{2}}{\left\|E E^{*}\left(v^{(k)}\right)\right\|^{2}+\left\|L E^{*}\left(v^{(k)}\right)\right\|^{2}}=\mu .
\end{gathered}
$$

Detto $\Gamma$ l'insieme, supposto non vuoto, dei vettori $w$ di $S_{B}^{(0)}$ per ognuno dei quali si ha:

$$
E^{*}(w) \subset S_{B}^{(0)}, \quad L^{*}(w)=\Omega, \quad \underset{B_{i}}{\operatorname{estr}, \text { inf. }} E_{i} E^{*}(w)>0, \quad \underset{F_{j}}{\text { estr. inf. } L_{j} E^{*}(w)}>0
$$

ed indicato con $F(w)$ il maggiore fra gli $n+m$ numeri:

$$
\underset{B_{i}}{\text { estr. sup. }} \frac{w_{i}}{E_{i} E^{*}\left(w_{i}\right)}, \quad \text { estr. sup. } \frac{-M_{j}(w)}{L_{j} E^{*}(w)},
$$

se sono soddisfatte le ipotesi $\left.1^{\mathrm{a}}\right), 2^{\mathrm{a}}$ ), $\left.3^{\mathrm{a}}\right)$, si ha:

estr. sup. $I(u) \leq$ estr. inf. $F(v)$.

$$
s-\omega
$$


Proviamo innanzitutto che, se $v$ è un vettore di $S \equiv S_{B}^{(0)}$ per il quale si ha: (9)

$$
L^{*}(v)=\Omega, \quad E^{*}(v) \subset S_{B}^{(0)}
$$

sussiste la diseguaglianza:

$$
\|v\|^{2}+\|M(v)\|^{2} \leq \mu^{2}\left(\left\|E E^{*}(v)\right\|^{2}+\left\|L E^{*}(v)\right\|^{2}\right) \text {. }
$$

Dato $\varepsilon>0$ arbitrario, sia $u_{\varepsilon}$ un vettore di $S$ tale che $\left\|L\left(u_{\varepsilon}\right)-M(v)\right\|<\varepsilon$. Tale vettore certo esiste in virtù della ipotesi $\left.3^{\text {a }}\right)$. Poichè $E\left(S_{B}^{(0)}\right) \supset S$, esisterà un vettore $u \subset S$ soluzione del seguente sistema:

$$
E(u)=v, \quad L(u)=-L\left(u_{\varepsilon}\right) .
$$

Tenendo presente la prima delle (9) e le (10), la (1) fornisce:

$$
\begin{gathered}
\left(u, E^{*}(v)\right)=\|v\|^{2}+\left(M(v), L\left(u_{\varepsilon}\right)\right)= \\
=\|v\|^{2}+\|M(v)\|^{2}+\left(M(v), L\left(u_{\varepsilon}\right)-M(v)\right)
\end{gathered}
$$

e quindi :

$$
\begin{gathered}
\|v\|^{2}+\|M(v)\|^{2} \leq\|u\|\left\|E^{*}(v)\right\|+\|M(v)\| \varepsilon \leq \\
\leq \mu\left(\|L(u)\|^{2}+\|E(u)\|^{2}\right)^{\frac{1}{2}}\left(\left\|E E^{*}(v)\right\|^{2}+\left\|L E^{*}(v)\right\|^{2}\right)^{\frac{1}{2}}+\|M(v)\| \varepsilon \leq \\
\left.\leq \mu\left(\|v\|^{2}+\|M(v)\|^{2}+\varepsilon^{2}+2 \varepsilon\|M(v)\|\right)^{\frac{1}{2}}\left(\| E E^{*}(v)\right)^{2}+\|\left. L E^{*}(v)\right|^{*}\right)^{\frac{1}{2}}+\|M(v)\| \varepsilon .
\end{gathered}
$$

Da ciò si deduce, per l'arbitrarietà di $\varepsilon$ :

$$
\|v\|^{2}+\|M(v)\|^{2} \leq \mu\left(\|v\|^{2}+\|M(v)\|^{2}\right)^{\frac{1}{2}}\left(\left\|E E^{*}(v)\right\|^{*}+\left\|L E^{*}(v)\right\|^{2}\right)^{\frac{1}{2}}
$$

cioè la diseguaglianza annunciata.

Per ogni $u \subset S_{B}^{(0)}$ consideriamo il vettore $v$ verificante le equazioni :

$$
E^{*}(v)=u, \quad L^{*}(v)=\mathbf{\Omega} .
$$

Anzichè considerare il funzionale $I(u)$ definito in $S_{B}^{(0)}-\omega$, potremo, come nella dimostrazione del teorema $I$, considerare il funzionale:

$$
J(v)=\frac{\left\|E^{*}(v)\right\|^{2}}{\left\|E E^{*}(v)\right\|^{2}+\left\|L E^{*}(v)\right\|^{2}},
$$

definito in $E^{*-1}\left(S_{B}^{(0)}\right)-\omega$. Detta $\left\{v^{(k)}\right\}$ la successione considerata nell' enunciato, facciamo vedere che:

$$
\lim _{k \rightarrow \infty}\left(\left\|v^{(k)}-\mu E E^{*}\left(v^{(k)}\right)\right\|^{2}+\left\|M\left(v^{(k)}\right)+\mu L E^{*}\left(v^{(k)}\right)\right\|^{2}\right)=0
$$

Si ha infatti :

$$
\begin{gathered}
\left\|v^{(k)}-\mu E E^{*}\left(v^{(k)}\right)\right\|^{2}+\left\|M\left(v^{(k)}\right)+\mu L E^{*}\left(v^{(k)}\right)\right\|^{2}= \\
=\left\|v^{(k)}\right\|^{2}+\left\|M\left(v^{(k)}\right)\right\|^{2}+2 \mu\left[\left(M\left(v^{(k)}\right), L E^{*}\left(v^{(k)}\right)\right)-\left(v^{(k)}, E E^{*}\left(v^{(k)}\right) \|\right]+\right. \\
+\mu^{2}\left[\left\|E E^{*}\left(v^{(k)}\right)\right\|^{2}+\left\|L E^{*}\left(v^{(k)}\right)\right\|^{2}\right]= \\
=\left\|v^{(k)}\right\|^{2}+\left\|M\left(v^{(k)}\right)\right\|^{2}-2 \mu\left\|E^{*}\left(v^{(k)}\right)\right\|^{2}+\mu^{2}\left(\left\|E E^{*}\left(v^{(k)}\right)\right\|^{2}+\left\|L E^{*}\left(v^{(k)}\right)\right\|^{*}\right) \leq \\
\left.\leq 2 \mu^{2}\left(\left\|E E^{*}\left(v^{(k)}\right)\right\|^{2}+\left\|L E^{*}\left(v^{(k)}\right)\right\|^{2}\right)-2 \mu\left\|E\left(v^{(k)}\right)\right\|^{2}\right) .
\end{gathered}
$$


Per la seconda delle condizioni b) esiste una costante $A>0$ tale che per ogni $k$ riesea :

$$
\left\|E E^{*}\left(v^{(k)}\right)\right\|^{2}+\left\|L E^{*}\left(v^{(k)}\right)\right\|^{2} \leq A .
$$

Cid implica la (11).

Sia ora $w$ un arbitrario vettore di $\Gamma$. Se qualcuna delle seguenti $n+m$ funzioni :

$$
\mu-\frac{w_{i}}{E_{i} E^{*}(w)}, \quad \mu+\frac{M_{j}(w)}{L_{j} E^{*}(v)}
$$

ha estremo inferiore nullo o negativo, riesce $F(w) \geq \mu$.

Sia infatti :

$$
\underset{B_{i}}{\operatorname{estr.inf} .}\left(\mu-\frac{w_{i}}{E_{i} E^{*}(w)}\right) \leq 0 \quad\left[\underset{G_{j}}{\operatorname{estr.inf} .}\left(\mu+\frac{M_{j}(w)}{L_{j} E^{*}(w)}\right) \leq 0\right] .
$$

Sarà allora :

$$
\mu \leq \underset{B_{i}}{\operatorname{estr.~sup.}} \frac{w_{i}}{E_{i} E^{*}(w)} \leq F(w) \quad\left[\mu \leq \underset{G_{j}}{\operatorname{estr.~sup.}} \frac{-M_{j}(w)}{L_{j} E^{*}(w)} \leq F(w)\right] .
$$

Orbene, mostreremo che è impossibile che, quali si siano $i$ ed $j$, riesca:

$$
\underset{B_{i}}{\operatorname{estr.inf}}\left(\mu-\frac{w_{i}}{E_{i} E^{*}(w)}\right)>0, \quad \text { estr. inf. }\left(\mu+\frac{M_{j}(w)}{L_{j} E^{*}(w)}\right)>0 .
$$

Ammettendo, per assurdo, che ciò si verifichi, si ha, detto $p^{2}$ un oppor. tuno numero positivo:

estr. inf. $\left(\mu E_{i} E^{*}(w)-w_{i}\right) \geq p^{2}>0, \quad$ estr. inf. $\left(\mu L_{j} E^{*}(w)+M_{j}(w) \geq p^{2}>0\right.$.

$B_{i}$

Sia $\left\{v^{(k)}\right\}$ la successione di vettori già considerata. Si ha:

$$
\begin{gathered}
\left(\mu E_{1} E^{*}(w)-w_{i}\right) E_{i} E^{*}\left(v^{(k)}\right)=v_{i}^{(k)} E_{i} E^{*}(w)-w_{i} E_{i} E^{*}\left(v^{(k)}\right)+\left(\mu E_{i} E^{*}\left(v^{(k)}\right)-v_{i}^{(k)}\right) E_{i} E^{*}(w) \\
\left(\mu L_{j} E^{*}(w)+M_{j}(w)\right) L_{j} E^{*}\left(v^{(k)}\right)=M_{j}(w) L_{j} E^{*}\left(v^{(k)}\right)-M_{j}\left(v^{(k)}\right) L_{g} E^{*}(w)+ \\
+\left(\mu L_{j} E^{*}\left(v^{(k)}\right)+M_{j}\left(v^{(k)}\right)\right) L_{j} E^{*}(w) .
\end{gathered}
$$

Da queste eguaglianze, tenendo presente che:

$$
\left(v^{(k)}, E E^{*}(w)\right)-\left(w, E E^{*}\left(v^{(k)}\right)\right)+\left(M(w), L E^{*}\left(v^{(k)}\right)\right)-\left(M\left(v^{(k)}\right), L E^{*}(w)\right)=0,
$$

si trae:

$$
\begin{gathered}
\sum_{i=1}^{n} \int_{B_{i}} E_{i} E^{*}\left(v^{(k)}\right) d \beta_{i}+\sum_{j=1}^{m} \int_{G_{j}} L E_{j} E^{*}\left(v^{(k)}\right) d \gamma_{j} \leq \frac{1}{p^{2}}\left[\left(\mu E E^{*}\left(v^{(k)}\right)-v^{(k)}, E E^{*}(w)\right)+\right. \\
+\left(\mu L E^{*}\left(v^{(k)}\right)+M\left(v^{(k)}\right), L E^{*}(w)\right] \leq \frac{1}{p^{2}}\left(\left\|v^{(k)}-\mu E E^{*}\left(v^{(k)}\right)\right\|^{\ell}+\right. \\
\left.+\left\|M\left(v^{(k)}\right)+\mu L E^{*}\left(v^{(k)}\right)\right\|^{2}\right)^{\frac{1}{2}}\left(\left\|E E^{*}(w)\right\|^{2}+\left\|L E^{*}(v)\right\|^{2}\right)^{\frac{1}{2}}
\end{gathered}
$$


e quindi l'assurdo:

$$
\lim _{k \rightarrow \infty}\left(\sum_{i=1}^{n} \int_{B_{i}} E_{i} E^{*}\left(v^{(k)}\right) d \beta_{i}+\sum_{j=1}^{m} \int_{G_{j}} L_{j} E^{*}\left(v^{(k)}\right) d \gamma_{j}\right)=0 .
$$

Analogo teorema può enunciarsi e dimostrarsi in sostituzione del teorema II allorchè non si ammetta l'esistenza del minimo di $I(u)$.

\section{Equazioni alle derivate parziali lineari del secondo ordine di tipo ellittico.}

Sia $A$ un campo (insieme aperto) limitato dallo spazio euclideo $S_{,}$. La frontiera $\mathfrak{F} A$ di $A$ sia costituita da un numero finito di ipersuperficie chiuse di classe due, cioe localmente rappresentabili con funzioni continue e dotate di derivate prime $\theta$ seconde continue. Sia $A^{\prime}$ un campo contenente $A+\mathcal{F} A$ e si consideri in $A^{\prime}$ l'operatore differenziale del secondo ordine di tipo ellittico positivo:

$$
E(u) \equiv \sum_{h, k}^{1, r} a_{h k} \frac{\partial^{2} u}{\partial x_{k} \partial x_{k}}+\sum_{k=1}^{r} b_{k} \frac{\partial u}{\partial x_{k}}+c u
$$

Le funzioni $a_{h k}$ siano di classe due (7) in $A^{\prime}$ ed ivi dotate di derivate seconde hölderiane. Le $b_{k}$ di classe uno con derivate prime hölderiane e la $c$ sia hölderiana in $A^{\prime}$ ed ivi mai positiva.

Vogliamo considerare un notevole caso particolare del teorema $I$.

Assumeremo $n=m=1 ; B_{1}=A ; G_{1}=\mathscr{F} A$. Come $S_{B}$ la varietà delle funzioni misurabili in $A$ ed ivi di quadrato integrabile secondo LEBESGUE. $S_{G}$ sarà la varietà delle funzioni definite su $\mathscr{F} A$ ed ivi di quadrato ipersuperficialmente integrabile secondo LEBESG UE. La varietà $S_{B}^{(0)} \equiv S$ sarà quella delle funzioni di classe uno in $A+\mathscr{F} A$ e dotate di derivate seconde continue e di quadrato integrabile in $A$.

Assumeremo la trasformazione $E(u)$ definita dalla (13) ed $E^{*}(u)$ al modo seguente:

$$
E^{*}(u)=\sum_{h, k}^{1, r} \frac{\partial^{2} a_{k k} u}{\partial x_{h} \partial x_{k}}-\sum_{k=1}^{r} \frac{\partial b_{k} u}{\partial x_{k}}+c u=0
$$

La $L(u)$ e la $L^{*}(u)$ si assumono coincidenti con la trasformazione che ad una funzione $u$ di $S$ fa corrispondere la sua traccia su $F A$ cambiata di segno.

La funzione d'insieme $\beta_{1}$ \& l'ordinaria misura in $S$, e la $\gamma_{1}$ la.misura ipersuperficiale su $\mathfrak{F} A$.

Fatte tali assunzioni, verifichiamo il sussistere delle ipotesi $\left.\left.\left.1^{a}\right), 2^{a}\right), 3^{a}\right)$.

(7) Dicendo ohe una data funxione è di classe $q$ in un insieme, intendo che ò ivi continua assieme alle sue derivate parziali fino a quelle di ordine $q$ incluso. 
La (1) dell'ipotesi $1^{\text {a }}$ ) ஓे verificata assumendo:

$$
M^{*}(u)=-\left(\frac{\partial u}{\partial v}+b u\right), \quad M(v)=-\frac{\partial v}{\partial v}
$$

dove si è posto, detta $n$ la normale ad $\mathscr{F} A$ interna ad $A$ :

$$
\frac{\partial}{\partial v} \equiv \sum_{h, k}^{1, r} a_{h k} \cos \left(x_{h}, n\right) \frac{\partial}{\partial x_{k}} ; \quad b=\sum_{h=1}^{r}\left(b_{h}-\sum_{k=1}^{n} \frac{\partial a_{k k}}{\partial x_{k}}\right) \cos \left(x_{h}, n\right) .
$$

Sussiste infatti per ogni coppia di funzioni di $S$ la ben nota formola di Grean :

$$
\int_{A}\left(u E^{*}(v)-v E(u)\right) d \tau=\int_{\mathscr{F} A}\left[v\left(\frac{\partial u}{\partial v}+b u\right)-u \frac{\partial v}{\partial v}\right] d \sigma
$$

che con le posizioni assunte altro non è che la (1).

Il ben noto teorema di unicità relativo ai due problemi:

$$
\begin{aligned}
& E(u)=v \quad \text { in } A, \quad u=U \text { su } \mathscr{F} A \\
& E^{*}(u)=v \text { in } A, \quad u=U \text { su } \mathscr{F} A,
\end{aligned}
$$

con $v$ continua in $A$ ed $U$ su $\mathscr{F} A$, assicura il sussistere dell'ipotesi $2^{a}$ ).

Infine, poichè appartengono ad $S$ i polinomi, essendo ben noto che, data una qualsiasi funzione $U$ di $S_{G}$ ed $\varepsilon>0$, esiste un polinomio $P$ tale che:

$$
\int_{\mathscr{F} A}|U-P|^{z} d \sigma<\varepsilon
$$

risulta verificata anche l'ipotesi $3^{a}$ ).

Più delicata è la verifica dell'ipotesi ammessa nel teorema I, relativa all' esistenza del massimo di $I(u)$. Più semplice si presenta invece il verificare le ipotesi del teorema VII.

Facciamo intanto vedere che:

$$
I(u)=\frac{\int u^{2} d \tau}{\int_{A}|E(u)|^{2} d \tau+\int_{\xi_{A}} u^{2} d \sigma}
$$

è limitato superiormente in $S-\omega$.

Sia $w(x)$ un'arbitraria funzione di classe uno in $A+\mathscr{F} A$ e di classe due in $A$ verificante le condizioni :

$$
\underset{A}{\text { estr. inf. } E^{*}(w)>0} \text { in } A, \quad w=0 \quad \text { su } \quad \mathfrak{F} A \text {. }
$$

Sia $u_{0}(x)$ la funzione verificante la $E\left(u_{0}\right)=0$ in $A$ e che su $\mathscr{F} A$ coincide con $u$ e sia $u_{1}=u-u_{0}$. 
Dalla (14), ponendo al posto di $u$, successivamente, $u_{0}^{2}$ ed $u_{1}^{2}$ e ricordando che $w$ mai positiva in $A$, si trae :

$$
\begin{aligned}
& \int_{A} u_{0}^{2} E^{*}(w) d \tau \leq-\int_{F_{A}} u_{0}^{2} \frac{\partial w}{\partial \nu} d \sigma, \\
& \int_{A} u_{1}^{2} E^{*}(w) d \tau \leq 2 \int_{A} u_{\mathfrak{i}} w E\left(u_{1}\right) d \tau
\end{aligned}
$$

e quindi :

$$
\begin{gathered}
\int_{A} u_{0}^{2} d \tau \leq \frac{\max _{\mathcal{F}_{A}}\left|\frac{\partial w}{\partial v}\right|}{\text { estr. inf. } E^{*}(w)} \int_{\mathcal{F}_{A}} u_{0}^{2} d \sigma \\
\int_{A} u_{1}^{2} d \tau \leq \frac{2 \max _{A}|w|}{\text { estr. inf. } E^{*}(w)} \int_{A}\left|E\left(u_{1}\right)\right|^{2} d \tau\left({ }^{8}\right) .
\end{gathered}
$$

Dalle (16), (17) segue la limitatezza di $I(u)$.

Diremo $\mu$ l'estremo superiore di $I(u)$. Se $u$ \& una qualsivoglia funzione di $S$, possono determinarsi due successioni di funzioni $\left\{f_{s}\right\}$ e $\left\{U_{s}\right\}$ rispettivamente definite in $A$ e su $\mathscr{F} A$, tali che:

$$
f_{s}>0, \lim _{s \rightarrow \infty} \int\left(f_{s}-|E(u)|\right)^{2} d s=0 ; \quad U_{s}<0, \lim _{s \rightarrow \infty} \int_{\mathscr{F} A}\left(U_{s}+|u|\right)^{2} d s=0
$$

e tali inoltre che la funzione $u_{s}$ verificante il sistema:

$$
E\left(u_{s}\right)=f_{s} \text { in } A, \quad u=U_{s} \text { su } \mathscr{F} A
$$

appartenga ad $S\left({ }^{\circ}\right)$.

Sarà allora:

$$
\lim _{s \rightarrow \infty} I\left(u_{s}\right) \geq I(u)
$$

(8) La (16) e la (17) sono state giă ottenute con analogo procedimento da CaccroppoLı (loc. cit. $\left({ }^{3}\right)$ ) assumendo però come $w$ la soluzione del sistema $E^{*}(v)=1$ in $A, w=0$ su $\mathscr{F} A$.

La possibilita di pervenire ad una maggiorazione quale la (16) era stata anche indicata - sia pure in un caso particolare - nella mia Nota: Sulla maggiorazione dell'errore di approssimazione nei procedimenti di integrazione numerica delle equazioni della Fisica Matematica, "Rend. Acc. delle Sei. Fis. Mat. e Nat. di Napoli , (1950).

(9) $\dot{\mathbf{H}}$ opportuno ricordnm che il sistema foi monomi $\left|m_{s}\right|$ is tale che il sistema di vettori $\left|\mathbb{E}\left(m_{s}\right), m_{s}\right|$ iे completo nollo spazio di Hurneser dei rettori arenti dun componenti, la prima definita in $A$, la seconda su $\mathcal{F} A$. 
Questa diseguaglianza ci permette di affermare che esiste una successione $\left\{u^{(k)}\right\}$ di funzioni di $S$ tali che:

$$
\lim _{k \rightarrow \infty} I\left(u^{(k)}\right)=\mu
$$

e per la quale si ha:

$$
E\left(u^{(k)}\right)>0 \quad \text { in } \quad A, \quad L\left(u^{(k)}\right) \equiv-u^{(k)}>0 \quad \text { su } \quad \mathscr{F} A .
$$

Sia $v^{(k)}$ la soluzione del sistema :

$$
E^{*}\left(v^{(k)}\right)=u^{(k)} \quad \text { in } \quad A, \quad L^{*}\left(v^{(k)}\right) \equiv-v^{(k)}=0 \quad \text { su } \quad \mathcal{F} A .
$$

La successione $\left\{v^{(k)}\right\}$ verifica le condizioni a) e c) dell' enunciato del teorema VII. Verifichiamo le b). A tale scopo occorre far ricorso alla funzione di Green $G(x, y)$ per il problema di DiRICHLEx in $A$, relativo all'operatore $E$. L' esistenza di tale funzione e le proprietà che ne sfrutteremo in seguito sono assicurate dalle ipotesi assunte sui coefficienti di $E$ e sulla frontiera di $\mathscr{F} A\left({ }^{10}\right)$.

Detta $\sigma$, la misnra ipersuperficiale della ipersfera unitaria, poniamo:

$$
k_{r}=\left\{\begin{array}{lll}
(r-2) \sigma_{r} & \text { per } & r>2 \\
2 \pi & \text { per } & r=2
\end{array} .\right.
$$

Diciamo $a(x) \equiv a\left(x_{1}, x_{2}, \ldots, x_{r}\right)$ il determinante della matrice $\left(a_{4 k}\right)$. In ogni punto $x$ di $A$ si ha:

$$
u(x)=\frac{1}{k_{v} \cdot \sqrt{a(x)} \int_{\mathscr{G A}}} u(y) \frac{\partial}{\partial v_{y}} G(x, y) d_{y} \sigma-\frac{1}{k_{r} \cdot \sqrt{a(x)}} \int_{A} E(u) G(x, y) d_{\nu} \tau .
$$

Poniamo :

$$
\begin{gathered}
u(y)=U(y) \quad \text { su } \quad \mathscr{F} A, \quad E(u)=v(y) \quad \text { in } A, \\
\frac{1}{k_{r} \sqrt{a(x)}} \frac{\partial}{\partial v_{\nu}} G(x, y)=H_{1}(x, y), \quad \frac{1}{k_{r} \sqrt{a(x)}} G(x, y)=H_{2}(x, y), \\
K_{14}(y, t)=\int_{A} H_{1}(x, y) H_{1}(x, t) d_{x} \tau, \quad K_{12}(y, z)=\int_{A} H_{1}(x, y) H_{z}(x, z) d_{x} \tau, \\
K_{22}(z ; s)=\int_{A} H_{2}(x, z) H_{2}(x, s) d_{x} \tau .
\end{gathered}
$$

(10) Cfr. G. Giraud, Sur le problème de Divichlet, ece., Ann. Ee. Sup. ^, t. 49, 1926, pag. 1.128; M. Gevrer, Determination et emploi des fonctions de Green ecc., Sour. de Math. ", t. 9, 1980. p. 1.80. Cfr. anche loc. cit. (1). 
Si ha:

$$
\begin{gathered}
\|u\|^{2}=Q(U, v)=\iint_{(\mathscr{F} A)^{2}} K_{t s}(y, t) U(y) U(t) d_{\nu} \sigma d_{t} \sigma+ \\
+2 \int_{\mathscr{F} A} d_{\nu} \sigma \int_{A} K_{12}(y, z) U(y) v(z) d_{z} \tau+\iint_{A^{2}} K_{22}(z, s) U(z) U(s) d_{z} \tau d_{a} \tau .
\end{gathered}
$$

Consideriamo lo spazio di HLLBerT $\mathscr{H}$ dei vettori a due componenti $U$ e $v$ la prima di quadrato ipersuperficialmente integrabile su $\mathscr{F} A$ e la seconda di quadrato integrabile in $A$. La forma quadratica $Q(U, v)$ definita in $\mathcal{H}$ o total. mente continua (1") essendo i nuclei $K_{11}(y, t), K_{12}(y, z), K_{22}(z, s)$ nuclei di FREDHoLm. Il funzionale:

$$
\frac{Q(U, v)}{\|U\|^{2}+\|v\|^{2}}
$$

è dotato di massimo $\left({ }^{12}\right)$ in $\mathcal{H}$ privato dell'origine. Tale massimo coincide con l'estremo superiore di $I(u)$ in $S$ (ofr. nota a piè di pagina $\left({ }^{9}\right)$ ),

Sfruttando proprietà più intime di $G(x, y)$, si potrebbe dimostrare che se $U_{n}, v_{0}$ è il vettore massimante del funzionale (18), esiste uua funzione $u_{\mathrm{n}}$ di $S$ tale che:

$$
u_{0}=U_{0} \quad \text { su } \quad \mathscr{F} A, \quad E\left(u_{0}\right)=v_{0} \quad \text { in } A
$$

e quindi che esiste il massimo di $I(u)$ in $S-\omega$. Cio però non è essenziale ai nostri scopi. Infatti possiamo supporre che la successione $u^{(k)}$ sopra considerata sia tale che:

$$
\lim _{k \rightarrow \infty} \int_{A}\left[E\left(u^{(k)}\right)-v_{0}\right]^{2} d \tau=\lim _{k \rightarrow \infty} \int_{\mathscr{J} A}\left[L\left(u^{(k)}\right)-U_{0}\right]^{2} d \sigma=0 .
$$

Essendo:

$$
H\left(v^{(k)}\right)=\int_{A}\left|E\left(u^{(k)}\right)\right| d \tau+\int_{\mathscr{d} A}\left|L\left(u^{(k)}\right)\right| d \sigma
$$

si ha allora:

$$
\begin{gathered}
\lim _{k \rightarrow \infty} H\left(v^{(k)}\right)=\int_{A}\left|v_{0}\right| d \tau+\int_{\mathcal{F}_{A}}\left|U_{0}\right| d \sigma>0 \\
\lim _{k \rightarrow \infty}\left(\left.\int_{A}\left|E E^{r}\left(v^{(k)}\right)_{\mid}^{2} d \tau+\int_{\mathscr{F}_{A}}\right| E^{*}\left(v^{(k)}\right)\right|^{2} d \sigma\right)=\int_{A} v_{0}^{2} d \tau+\int_{\mathscr{F}_{A}} U_{0}^{2} d \sigma<+\infty .
\end{gathered}
$$

È perciò verificata la b).

(11) Cfr. loc. cit. (1), pag. 219.

(12) Cfr. loc. cit. (1), pag. 220. 


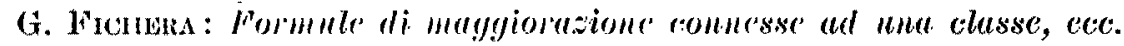

Possiamo allora applicare al caso particolare considerato il teorema VII, deducendo il seguente:

VIII. - Sia $w(x)$ un'arbitraria funzione definita in $A+\mathscr{F} A$, appartenente ad $S$ assieme ad $E^{*}(w)$ e verificante le condizioni:

$$
\begin{gathered}
w=0 \quad \text { su } \quad \mathfrak{F} A, \\
E^{*}(w)<0 \quad \text { su } \quad \mathscr{F} A, \\
\text { estr. inf. } E E^{*}(w)>0 .
\end{gathered}
$$

Nelle ipotesi ammesse per $A$ e per l'operatore del secondo ordine di tipo ellittico $E$, qualunque sia la funzione $u(x)$ di classe uno in $A+\mathfrak{F} A$ e dotata di derivate seconde continue $e$ di quadrato integrabile in $A$ si ha:

$$
\int_{A} u^{2} d \tau \leq F(w)\left\{\int_{A}|E(u)|^{2} d \tau+\int_{\mathscr{E} A} u^{2} d \sigma\right\}
$$

essendo $F(w)$ il maggiore dei due numeri:

$$
\text { estr. sup. } \frac{w}{E E^{*}(w)}, \quad \max _{\sharp A} \frac{-\frac{\partial w}{\partial v}}{E^{*}(w)}
$$

(v conormale interna a $\mathfrak{F A}$ ).

Con procedimento analogo ed anzi con maggiore semplicità dal teorema VII si traggono i due seguenti:

IX. - Sia $w(x)$ una funzione verificante tutte le ipotesi specificate nel teorema precedente, ma in luogo della (21) la seguente:

$$
E E^{*}(w)=0 \text { in } A .
$$

Qualunque sia la soluzione $u_{0}(x)$ di classe uno in $A+\mathscr{F} A$ dell' equazione $E\left(u_{0}\right)=0$, si ha:

$$
\int_{A} u_{0}^{2} d \tau \leq\left(\max _{\mathcal{F} A} \frac{-\frac{\partial w}{\partial \nu}}{E^{*}(w)}\right) \int_{\mathscr{G} A} u_{0}^{2} d \sigma
$$

X. - Sia $w(x)$ una funzione verificante tutte le ipotesi del teorema VIII, ma in luogo della (20) la seguente:

$$
E^{*}(w)=0 \quad \text { su } \quad \mathscr{F} A .
$$


Qualunque sia la funzione $u_{1}(x)$ di classe uno in $A+g A$, dotata di derivate seconde coutinue e di quadrato integrabile in $A$ e nulla $s u \mathscr{F} A$, si ha:

$$
\int_{A} u_{i}^{2} d \tau \leq\left(\text { estr. sup. } \frac{w}{E E^{*}(w)}\right) \int_{A}\left|E\left(u_{1}\right)\right|^{2} d \tau \text {. }
$$

La (24) e la (26) sono del tipo rispettivamente della (16) e della (17). Occorre però notare ohe l'applicazione delle (22), (24). (26) conduce a maggiorazioni più strette che non le analoghe deducibili da (16), (17).

Ciò è di grande importanza nei problemi quantitativi ad esempio per la maggiorazione dell' errore nel classico metodo di calcolo delle soluzioni del sistema:

$$
E(u)=f \quad \text { in } A, \quad u=g \text { su } \mathscr{F} A,
$$

cosidetto dei minimi quadrati.

Dato un sistema $\left\{m_{s}\right\}$ di funzioni tali che il sistema di vettori $\left\{E\left(m_{s}\right), m_{s}\right\}$ sia completo in $\mathcal{H}$, si determinino le costanti $c_{s}^{(n)}$ mediante la condizione:

$$
\int\left[f-\sum_{s=1}^{n} c_{s}^{(n)} E\left(m_{s}\right)\right]^{2} d \tau+\int\left[g-\sum_{s=1}^{n} c_{s}^{(n)} m_{s}\right]^{2} d \sigma=\text { minimo. }
$$

Dalla (22) si deduce che la successione $\sum_{s=1}^{n} c_{s}^{(n)} m_{s}$ converge in media verso la soluzione di (27), nonchè una maggiorazione per l'errore quadratico medio

$$
\int\left[u-\sum_{s=1}^{n} c_{s}^{(n)} m_{s}\right]^{2} d \tau
$$

亡̀ evidente l'interesse pratico di avere per $F(w)$ un valore quanto più piccolo è possibile. ellittico :

Sia $r=2$ ed $E(u) \equiv \Delta_{2} u \equiv \frac{\partial^{2} u}{\partial x_{1}^{2}}+\frac{\partial^{2} u}{\partial x_{2}^{2}}$. Assumiamo per $A$ il dominio

$$
\frac{x_{1}^{2}}{a^{2}}+\frac{x_{2}^{2}}{b^{2}} \leq 1 \quad(a \geq b>0) .
$$

Per conseguire la (22) si può considerare la funzione:

$$
w=\left(\frac{x_{1}^{2}}{a^{2}}+\frac{x_{2}^{2}}{b^{2}}\right)^{2}-8\left(\frac{x_{1}^{2}}{a^{2}}+\frac{x_{2}^{2}}{b^{2}}\right)+7
$$

per modo che $F(w)$ sarà il maggiore dei due numeri :

$$
\frac{7 a^{4} b^{4}}{24\left(a^{4}+b^{4}\right)+16 a^{2} b^{2}}, \quad \frac{3 a^{2} b}{a^{2}+3 b^{2}} .
$$


Si noti che per $r=2$ si può concedere al campo $A$ una maggiore generalità e, ad esempio, supporre che la sua frontiera sia costituita da archi di curva di classe due che si saldano senza formare cuspidi.

Tutte le premesse occorse per stabilire i teoremi VIII, IX, X risultano verificate anche in tali ipotesi per $\mathscr{F} A$.

Se $A$ e il dominio rettangolare:

$$
-a \leq x_{1} \leq a, \quad-b \leq x_{2} \leq b
$$

ed $E(u) \equiv \Delta_{2} u$, si assuma :

$$
w=\left(x^{2}-a^{2}\right)\left(y^{2}-b^{2}\right)
$$

e quindi $F(w)$ e il maggiore dei tre numeri :

$$
a, \quad b, \quad \frac{a^{2} b^{2}}{8} .
$$

Si noti che le (16), (17) pur fornendo maggiorazioni che - in genere saranno assai più grossolane di quelle date in (22), (24), (26), trttavia richiedono, per essere conseguite, un numero assai minore di informazioni sull'operatore $E$. Precisamente non occorre servirsi dell'esistenza della funzione di Greme e della soluzione del problema di Dirichler per tale operatore, sia pure con dati particolari, ma soltanto di quella di qualche fnnzione verificante le (15). Ciò è da tener presente per l'impiego di formule di maggiorazione del tipo di quelle in questione a scopi non quantitativi, ma, per esempio, soltanto esistenziali $\left({ }^{43}\right)$.

Il sistema (6), nel caso particolare considerato nel presente paragrafo, $\otimes$ il seguente:

$$
\left\{\begin{array}{l}
v_{0}-\mu E E^{*}\left(v_{0}\right)=0 \text { in } \quad D, \\
\frac{\partial v_{0}}{\partial v}+\mu E^{*}\left(v_{0}\right)=0 \text { su } \mathscr{F} D
\end{array} \quad\left(v_{0}=0 \text { su } \mathscr{F} D\right) .\right.
$$

Se esiste il massimo di $I(u)$, coincide con il più grande autovalore di questo sistema $\left({ }^{14}\right)$.

(13) Cfr. G. Stampacchia, Problema di Dirichlet e proprietà qualitative della soluzione, - Giornale di Matematiche di Battaglini *; S. IV, v. LXXX, f. 2॰, pp. 226.237.

(14) La Sig.na L. Pesci nella sua Tesi di laurea, compinta presso l'Istituto Nazionale per le Applicazioni del Calcolo, usando i procedimenti della presente Memoria, ha, fra l'altro, considerato il caso $r=1, E=\frac{d^{2}}{d x^{2}}, D \equiv(-c$, c). In tal caso nella (22) si può assu- 
3. Equazioni alle derivate parziali lineari del secondo ordine di tipo parabolico.

Sia $A$ il campo di $S_{r}$ considerato nel paragrafo precedente ed $E(u)$ l'operatore di tipo ellittico già considerato.

Indicherò con $D$ il dominio dello spazio $S_{r+1}$ delle variabili $x_{1}, x_{2}, \ldots$, $x_{r}, t$ definito da:

$$
x \equiv\left(x_{1}, x_{2}, \ldots, x_{r}\right) \subset A+\mathscr{F} A, \quad 0 \leq t \leq t_{0} .
$$

Considererò in $D$ i due operatori del secondo ordine di tipo parabolico:

$$
P(u)=E(u)-\frac{\partial u}{\partial t}, \quad P^{*}(u)=E^{*}(u)+\frac{\partial u}{\partial t} .
$$

Denoterò con $\mathscr{F}_{1} D, \mathscr{F}_{2} D, \mathscr{F}_{3} D$ le parti della frontiera $\mathscr{F} D$ di $D$ cosí definite:

$$
\begin{array}{lll}
\left.\mathfrak{F}_{1} D\right) & x \subset A, & t=0, \\
\left.\mathfrak{F}_{2} D\right) & x \subset \mathscr{F} A, & 0 \leq t \leq t_{0}, \\
\left.\mathfrak{F}_{3} D\right) & x \subset A, & t=t_{0} .
\end{array}
$$

Siano $u(x, t) \Theta v(x, t)$ una coppia di funzioni appartenenti all'insieme $S$ delle funzioni di classe uno in $D$ e dotate delle derivate seconde rispetto alle coordinate di $x$ continue in $D-\mathscr{F} D$ e di quadrato sommabile in $D$. Per esse sussiste la seguente formula di reciprocità :

$$
\begin{gathered}
\int_{0}^{t_{0}} d t \int_{A}\left[u P^{*}(v)-v P(u)\right] d \tau=\int_{0}^{t_{0}} d t \iint_{\mathscr{F} A}\left[v\left(\frac{\partial u}{\partial v}+b u\right)-u \frac{\partial v}{\partial v}\right] d \sigma- \\
-\int_{\mathscr{F}_{1} D} u v d \tau+\int_{\mathscr{F} D} u v d \tau .
\end{gathered}
$$

La direzione orientata $\vee$ e $b$ sono definiti come in precedenza.

mere $w=x^{4}-6 c^{2} x^{2}+5 c^{4}+c c^{2}(\rho>0)$, per modo che $F(v)$ è il maggiore fra $\mathrm{i}$ due numeri $\frac{c^{2}\left(5 c^{2}+p\right)}{24}, \frac{c\left(4 c^{2}+p\right)}{p}$ e quindi :

$$
\int_{-c}^{c} u^{2} d x \leq F^{\prime}(w)\left\{[u(c)]^{2}+[u(-c)]^{2}+\int\left(\frac{d^{2} u}{d x^{2}}\right)^{2} d x\right\}
$$

Il sistema $\left(6^{\prime}\right)$ nel caso attuale può scriversi (ponendo $\mu=\lambda^{-4}$ ed assunto $c=1$ ):

$$
\frac{d^{4} v_{0}}{d x^{4}}-\lambda^{4} v_{0}=0 ; \quad v(-1)=v(1)=0 ; \quad v^{\prime \prime}(-1)+\lambda^{4} v^{\prime}(-1)=0, v^{\prime \prime}(1)-\lambda^{4} v^{\prime}(1)=0 .
$$

Il più grande autovalore $\mu$ (corrispondente al più piccolo $\lambda$ ) si calcola in tal caso elementarmente ed è $\simeq 1.13$. Il minimo valore di $F(w)$ al variare di $p$ in $(0,+\infty)$ è $\simeq 1.17$. 
Il funzionale che prenderemo in considerazione è il seguente :

$$
I(u)=\frac{\int_{0}^{t_{0}} d t \int_{A} u^{2} d \tau}{\int_{0}^{t_{0}} d t \int_{A}|P(u)|^{2} d \tau+\int_{\mathscr{F}_{1} D} u^{2} d \tau+\int_{0}^{t_{0}} d t \int_{\mathcal{F}_{A}} u^{2} d \sigma} .
$$

Detto $\omega$ l'elemento nullo di $S$, ciò la funzione identicamente nulla in $D$, dimostreremo che $I(u)$ definito in $S-\omega$ è ivi limitato. Sia $w$ una funzione appartenente ad $S$ e verificante le condizioni :

$$
\text { estr. inf. } P^{*}(w)>0 \text { in } D-\mathscr{F} D, \quad w=0 \text { su } \mathscr{F}_{2} D+\mathscr{F}_{3} D .
$$

Sia $u_{1}$ una funzione di $S$ nulla sa $\mathscr{F}_{1} D+\mathscr{F}_{2} D$. Dalla (28) si trae per $u=u_{1}^{2} \otimes v=w$ :

$$
\int_{0}^{t_{0}} d t \int_{A} u_{1}^{2} P^{*}(w) d \tau \leq 2 \int_{0}^{t_{0}} d t \int_{A} u_{1} w P\left(u_{1}\right) d \tau .
$$

Se \& $u_{0} \subset S$ riesce $P\left(u_{0}\right)=0$, la (28) fornisce :

$$
\int_{0}^{t_{0}} d t \int_{A} u_{0}^{2} P(w) d \tau \leq-\int_{0}^{t_{0}} d t \int_{\mathcal{F}^{2} A} u_{0}^{2} \frac{\partial w}{\partial v} d \sigma-\int_{\mathscr{F}_{1} D} u_{0}^{2} w d \tau .
$$

Si trae quindi :

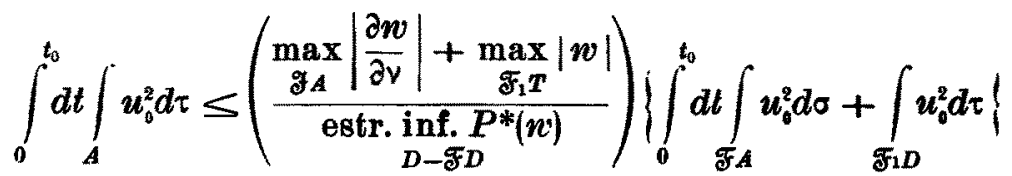

$$
\begin{aligned}
& \int_{0}^{t_{0}} d t \int_{A} u_{1}^{2} d \tau \leq\left(\frac{2 \max _{D}|w|}{\text { estr. inf. } P^{*}(w)}\right) \int_{0}^{t_{0} D} d t \int_{A}\left|P\left(u_{i}\right)\right| d \tau\left({ }^{15}\right) .
\end{aligned}
$$

Da queste disuguaglianze segue la limitatezza di $I(u)$.

Si può ora procedere come nel caso dell'operatore ellittico $E$, dato che anche per il problema:

$$
P(u)=f(x, t) \quad \text { in } \quad T-\mathscr{F} D, \quad u=g \quad \text { su } \quad \mathscr{F}_{1} D+\mathscr{F}_{2} D
$$

(15) Le (30), (31) sussistono anche se i coeffieienti doll'opentore $E$ dipendono da $t$, purchè si ammetta esistente la $w$ verificante le (29). 
non dipendendo i coefficienti di $E(u)$ da $t$ è già acquisita l'esistenza della funzione di GreEN $G(x, t ; y, s)$ che verifica le ipotesi richieste per applicare i ragionamenti fatti nel paragrafo precedente $\left({ }^{10}\right)$.

Si perviene cosi al seguente teorema.

XI. - Sia $w(x, t)$ una arbitraria funzione di classe uno nel cilindro $D$, appartenente ad $S$ assieme a $P^{*}(w)$ e verificante le condizioni:

$$
\begin{aligned}
& w=0 \quad \text { su } \quad \mathscr{F}_{z} D+\mathscr{F}_{3} D, \\
& \text { estr. inf. } P P^{*}(w)>0 \text { in } D \text {, } \\
& P^{*}(w)<0 \quad \text { su } \quad \mathscr{F}_{1} D+\mathscr{F}_{2} D .
\end{aligned}
$$

Indicato con $F(w)$ il maggiore fra $i$ tre numeri:

$$
\text { estr. sup. } \frac{w}{P P^{*}(w)}, \quad \max _{\mathscr{F}_{1} D} \frac{-w}{P^{*}(w)}, \quad \max _{\mathscr{F}_{2} D} \frac{-\frac{\partial w}{\partial v}}{P^{*}(w)} .
$$

(v conormale interna alla sezione retta $A+\mathcal{F} A d i D)$

sussiste per ogni $u$ di $S$ la seguente formola di maggiorazione:

$$
\int_{0}^{t_{0}} d t \int_{A} u^{2} d \tau \leq\left. F(w)\left|\int_{0}^{t_{0}} d t \int\right| P(u)\right|^{2} d \tau+\int_{0}^{t_{0}} d t \int_{\mathfrak{F} A} u^{2} d \sigma+\int_{\mathfrak{F}_{1} D} u^{2} d \tau \mid
$$

Sussistono anche gli analoghì dei teoremi IX e X. L'enunciazione di essi è ovvia.

Sia $r=1, A$ l'intervallo $(0, l)$ dell' asse $x$ e $P(u)=\frac{\partial^{2} u}{\partial x^{2}}-\frac{\partial u}{\partial t}$.

Si può assumere:

$$
w=\left[3 t_{0}^{2}\left(t-t_{0}\right)-\left(t-t_{0}\right)^{3}\right]\left(x^{2}-l x\right),
$$

per modo che $F(w)$ sarà il maggiore fra i tre numeri :

$$
\frac{1}{2} t_{0}^{2}, \quad \frac{1}{8} l^{2}, \quad \frac{1}{2} l .
$$

(16) Ofr. E. Rотнв, Über die Wärmeleitung mit nichtkostanten Koeffizienten in räum. lichen Falle, * Math. Ann. *, 102, (1930), pp. 650.670; Über die Grundlösung bei parabolischen Gleichungen, “ Math, Zeit. " 38, (1931), pp. 488-504.

G. GIRAUD, Sur certaines operations aux dérivées partielles du type parabolique, « $\mathrm{C} . \mathrm{R}$. Ac. Sci. », 195, (19332), pp. 98-100.

M. Gnvrey, Sur les equations aux dérivées partielles du type parabolique, "Jour. do Math. s, (6), 9, (1913), pp. 305.371 e (6), 10, (1914), pp. 105-148.

Cfr, anche loc. cit. (1). 
Si ha in tal caso:

$$
\begin{aligned}
\int_{0}^{t_{0}} d t \int_{0}^{l}|u(x, t)|^{2} d x & \leq F(w)\left\{\int_{0}^{t_{0}} d t \int_{0}^{l}\left|\frac{\partial^{2} u}{\partial x^{2}}-\frac{\partial u}{\partial t}\right|^{2} d x+\int_{0}^{l}|u(x, 0)|^{2} d x+\right. \\
& \left.+\int_{0}^{t_{0}}\left(|u(0, t)|^{2}+|u(l, t)|^{2}\right) d t\right\}
\end{aligned}
$$

\section{Altre applicazioni.}

Analoghe applicazioni - sia per le equazioni ellittiche, che per quelle paraboliche - possono farsi considerando problemi al contorno più generali. La condizione che sostanzialmente è richiesta per l'applicazione del metodo, come si constata dalla dimostrazione del teorema VIII, è la positività nel* l'interno del dominio della funzione di GREEN relativa al problema al con. torno che si considera. Così pure il metodo può applicarsi ad equazioni di ordine superiore al secondo con dati al contorno che però diano luogo ad una funzione di Grees positiva. Ad esempio si consideri l'equazione:

$$
\Delta_{2} \Delta_{2} u=f \text { in } A \quad\left(\Delta_{2}=\sum_{k=1}^{r} \frac{\partial^{2}}{\partial x_{k}^{2}}\right)
$$

con le condizioni al contorno:

$$
u=g_{1} \quad \text { su } \quad \mathscr{F} A, \quad \frac{\partial u}{\partial n}=g_{2} \quad(n \text { normale interna a } \mathfrak{F} A) .
$$

Sia $A$ un campo per il quale la funzione di GREeN è positiva, ad esem. pio un campo ipersferico $\left({ }^{17}\right)$.

Detta allora $w$ una funzione verificante le seguenti condizioni :

$$
w=\frac{\partial w}{\partial n}=0 \text { su } \mathscr{F} A, \quad \Delta_{2}^{2} w>0 \text { su } \mathscr{F} A \quad \frac{\partial \Delta_{2}^{2} w}{\partial n}>0 \text { su. } \mathscr{F} A, \quad \underset{A}{\text { estr. inf. } \Delta_{2}^{4} w>0}
$$

e indicato con $F(w)$ il maggiore fra i numeri :

$$
\underset{A}{\text { estr. inf. }} \frac{w}{\Delta_{2}^{4} w}, \quad \max _{\mathcal{F}_{A} A} \frac{\frac{\Delta_{2} w}{\partial \Delta_{2}^{2} w}}{\partial n}, \quad \max _{\mathcal{F}_{A}} \frac{-\frac{\partial \Delta_{2} w}{\partial n}}{\Delta_{2}^{2} w}
$$

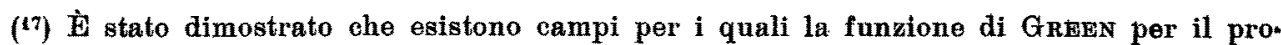
blema (32) (33) è di segno variabile. Cfr. P. Garabedian, A Partial Differential Equation Arising in Conformal Mapping, "Pacific Journ. of Math. ", v. I, n. 4. pp. 485.524. C. H. Lonw* NER - G. SzEGö, On Generation of Solutions of the Biharmonic Equation etc., * Dept. of Math. "Stanford University, Technical Report n. 13. 
sussiste la diseguaglianza :

$$
\int_{A} u^{2} d \tau \leq F(w)\left\{\int_{A}\left(\Delta_{2} \Delta_{2} u\right)^{2} d \tau+\int_{\mathcal{E}_{A}}\left|u^{2}+\left(\frac{\partial u}{\partial n}\right)^{2}\right| d \sigma\right\}
$$

I procedimenti seguiti possono anche applicarsi ad equazioni integrali o ad equazioni algebriche per problemi di calcolo di autovalori. In tal caso si vengono a ritrovare teoremi già sostanzialmente noti $\left({ }^{15}\right)$, che pertanto vengono inquadrati nella teoria generale da noi svolta.

(18) Cfr. J. Banta, Comptes Rendus s, 204, 472 (1937).

L. Collatz, Rigenwerte aufgaben, Akademische Verlaggesellschaft, (1949, Leipzig.

R. J. Dufris, Lover bounds for Eigenvalues, - The Phy. Rev. ", (1947), v. 71, pag. 827. 